\title{
Performance Analysis of IEEE802.16e Random Access Protocol with Mobility
}

\author{
Sang-Sik Ahn ${ }^{1}$, Hyong-Woo Lee ${ }^{1}$, Jun-Bae $\mathrm{Seo}^{2}$, and Choong-Ho Cho ${ }^{3}$ \\ ${ }^{1}$ Department of Electronics and Information Engineering, \\ Korea University \\ sahn@korea.ac.kr, hwlee@korea.ac.kr \\ ${ }^{2}$ Department of Wireless System Research, \\ Electronics and Telecommunications Research Institute, \\ 161 Gajeong-dong, Yuseong-gu, Daejeong 305-350, South-Korea \\ jbseo@etri.re.kr \\ ${ }^{3}$ Department of Computer Science, Korea University, \\ Chochiwon, Chungnam 339-700, South-Korea \\ chcho@korea.ac.kr
}

\begin{abstract}
In this paper, the performance of IEEE802.16e random access protocol with handover procedure is examined in terms of access throughput and mean access delay, by using equilibrium point analysis(EPA). In the analysis, retransmission probability, which is a typical input parameter in the literature so far, is iteratively obtained from equilibrium number of backlogs in the system in conjunction with a binary exponential backoff algorithm. In numerical examples, the effects of SSs' mobility on access throughput and mean access delay are examined.
\end{abstract}

\section{Introduction}

Among the various features of the physical layer in IEEE802.16a/b/c/d/e, we focus on orthogonal frequency-division-multiplexing(OFDM) with TDD mode. The frame structure and its detailed description of our interest are given in [1]-[3]. In this paper, we examine the performance of IEEE 802.16e MAC protocol with mobility by using EPA, since the analysis using a Markov chain to describe various states of a subscriber station(SS) is formidable [3] due to the explosion of the state space. One can find some previous works on IEEE802.16 random access protocol [3]-[6] in the literature. This paper is organized as follows. In section 2, the handover procedure of IEEE802.16e MAC protocol is described and its analysis is given. The numerical examples are discussed in section 3. Concluding remarks are given in section 4 .

\section{IEEE 802.16 MAC Protocol}

\subsection{Procedure of Bandwidth Request and Handover Ranging}

The random access protocol of IEEE802.16e is a class of demand-assigned multiple access(DAMA). The basic procedure of the random access protocol without handover 
procedure is summarized in [3]. Here, we focus on the handover procedure $(\mathrm{HO})$ only. Whenever an SS crosses a cell-boundary irrespective of its actions, such as, retransmissions of bandwidth request code, data transmission or waiting for CDMA allocation message, and so on, it performs the following handover procedure. According to the carrier-to-interference-noise ratio(CINR) of a serving base station(BS), an SS sends $\mathrm{HO}$-request message and receives HO-response message. After that, the SS sends HOindication message to the serving BS. At this point, the SS doesn't scan DL/UL-MAPs of the serving BS any more. Note that HO-request and HO-indication messages from the SS are also delivered through the random access procedure in parallel with data traffic transmission. Here, we assume that these two signalling messages are negligible for our modelling, because of their parallel transmission structure. In a target BS, the SS performs a HO-ranging procedure which is contention-based synchronization(or adjustment) to the system. It uses a PN code in $\mathrm{HO}$ ranging code group. Note that an $\mathrm{SS}$ has already known a group of $\mathrm{HO}$ ranging codes in a target BS from the neighbor cell advertisement message in the previous serving cell. At the end of HO-ranging procedure, registration, authentication and other procedures may follow or be omitted according to the $\mathrm{HO}$ optimization field within $\mathrm{HO}$ ranging response message. In case of an omission of such signalling procedures, the information of an SS is transferred to the target BS from the serving BS. The detailed operations are given in [2]. In order to complete $\mathrm{HO}$ procedures, the SS can restart the bandwidth ranging procedure. Therefore, the time before restarting the bandwidth ranging procedure in a target BS from the transmission termination in the previous serving BS forms a random delay at least due to the $\mathrm{HO}$ ranging procedure, if we may view the initiation epoch of $\mathrm{HO}$ as the beginning of HO-indication message transmission. For simplicity of the analysis, we assume that the elapsed time up to the bandwidth ranging procedure in a target BS from the $\mathrm{HO}$ ranging procedure, which includes signalling delay, takes $y$ frames.

\subsection{Equilibrium Point Analysis}

In TDD mode, it is hard to transmit a response message on DL-subframe in the $(i+1)$ th frame when the message corresponding to the response message has been received on UL-subframe in the $i$-th frame, because the decoding time for the message received from UL-subframe and the encoding time for the message to transmit on DL-subframe may overlap for practical implementation. We assume that the delay from the reception of the bandwidth request code to transmission of its response, i.e., processing delay, and the delay from the reception of the bandwidth request message to transmission of its channel allocation, i.e., scheduling delay, are respectively $z$ and $x$ frames. In addition, the delay by $\mathbf{T 3}$ timer on retransmissions and the delay of the HO procedure are respectively assumed to be $w$ and $y$ frames.

In Fig.1, a set of modes an SS can be in is shown. The modes C, B, R and T respectively denote the initial bandwidth request code transmission-, its retransmissions-, bandwidth request message transmission- and data transmission-mode. Additionally, $\mathrm{P}_{T i}, \mathrm{P}_{B j}, \mathrm{P}_{R k}$ and for $0 \leq i \leq z-1,0 \leq j \leq x-1$ and $0 \leq k \leq w-1$, denote the delay experienced by $\mathrm{C}, \mathrm{R}$ and $\mathrm{B}$ mode. The modes of $\mathrm{H}$ and $\underline{\mathrm{H}}_{l}$ for $0 \leq l \leq$ $y-1$ denote the $\mathrm{HO}$ ranging procedure with transition probability, $p_{h}$, and signalling 


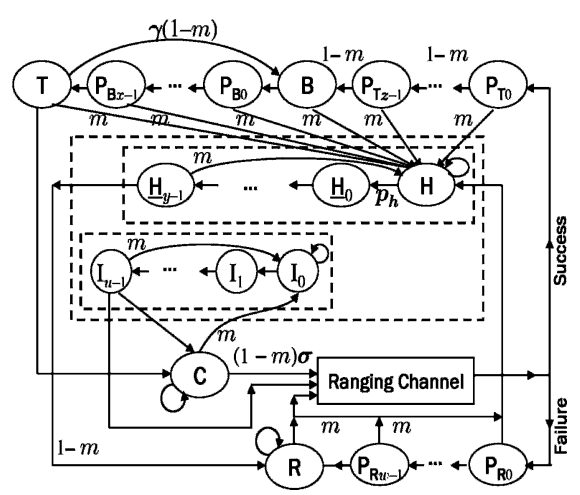

Fig. 1. A model of IEEE802.16e MAC protocol

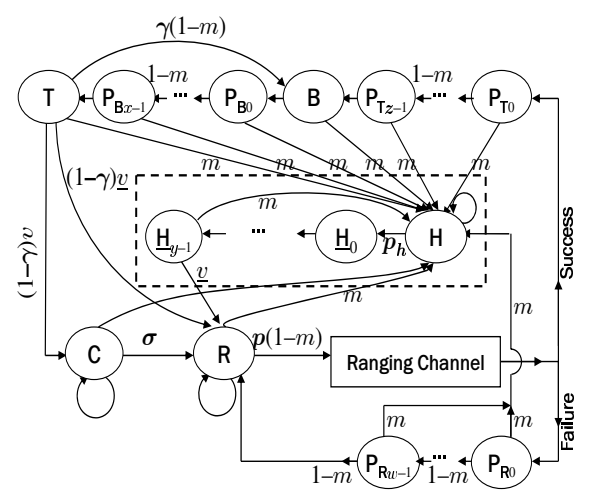

Fig. 2. A modified model of IEEE802.16e MAC protocol

delay. Finally, the mode, $I_{n}$, for $0 \leq n \leq u-1$, denotes that an SS moves out of a serving BS between traffic arrivals. Transition from one mode to another occurs at the end of a frame. In mode C, an SS has data to be transmitted with probability $\sigma$. In mode $\mathrm{R}$, an SS retransmits the bandwidth request code with probability $p$, after it is known that the previous transmission of a bandwidth request code is not successful. The transition from $\mathrm{T}$ to $\mathrm{B}$ indicates the piggyback of the bandwidth request message at the end of data transmission with probability $\gamma$. In each mode, it may move out of a serving BS with probability $m$. Once an SS enters the mode of $\underline{\mathrm{H}}_{0}$, its transitions to $\underline{\mathrm{H}}_{l}$ for $1 \leq l \leq y-1$ occur with probability one. We assume a finite population of $M \mathrm{SSs}$ in a given cell. Before proceeding further, we modify the model in Fig. 1 under the condition of $\sigma \leq p$ as shown in Fig. 2 in order to merge two inputs to the ranging channel into one [7]. In order to focus on the effect of mobility upon traffic transmission, the mode, $I_{n}, \forall n$, are ignored. The parameters, $v$ and $\underline{v}$, in Fig.2 are expressed as $v=\widehat{m}(1-\sigma / p)$, $\underline{v}=\widehat{m}(\sigma / p)$ and $\widehat{m}=1-m$. Accordingly, in a given BS, the system state is described by the vector $\left(C, R, B, T, P_{T i}, P_{B j}, P_{R k}, H, \underline{H}_{l}\right), \forall i, \forall j, \forall k$ and $\forall l$, where $C$ is the number of SSs in mode $\mathrm{C}, R$ is the number of SSs in mode $\mathrm{R}$, and so on. We denote the equilibrium variables of the state variables for the system by the corresponding lowercase letters, $\left(c, r, b, t, p_{T i}, p_{B j}, p_{R k}, h, \underline{h}_{l}\right), \forall i, \forall j, \forall k$ and $\forall l$. Since the transitions within the states to represent the delay, $p_{T i}, p_{B j}, p_{R k} \forall i, \forall j$ and $\forall k$, occur with probability $1-m$, one can readily find the following relations $: p_{T i+1}=\widehat{m} p_{T i}=\widehat{m}^{i+1} p_{T 0}$, $p_{B j+1}=\widehat{m}^{j+1} p_{B 0}$ and $p_{R k+1}=\widehat{m}^{k+1} p_{R 0}, \forall i, \forall j, \forall k$. At the states, $P_{T 0}, P_{R 0}, P_{B 0}, B$ and $T$, one can also obtain the followings : $p_{T 0}=S(r), p_{R 0}=p \widehat{m} r-S(r), p_{B 0}=\widehat{m} b$, $b=\widehat{m}\left(p_{T z-1}+\gamma t\right)$ and $t=\widehat{m} p_{B x-1}$, where $S(r)$ is the expected input to the system, which will be derived later. By the same way, at the states, $\underline{H}_{l}$ and $H$, one can get the followings : $\underline{h}_{l+1}=\underline{h}_{l}, \forall l, \underline{h}_{0}=p_{h} h$ and $h=m\left(\underline{h}_{y-1}+r+b+t+\breve{d}\right)+\left(1-p_{h}\right) h$, where $\breve{d}=\sum_{i=0}^{z-1} p_{T i}+\sum_{j=0}^{x-1} p_{B j}+\sum_{k=0}^{w-1} p_{R k}$. Because the sum of SSs in all the states must be $M$, the following equation is satisfied. 


$$
(1-\gamma) v t=\sigma\left(M-r-t-b-\check{d}-\sum_{l=0}^{y-1} \underline{h}_{l}-h\right)
$$

With some manipulations, one can express $b, t$ and each sum of $p_{T i}, p_{B j}$ and $p_{R k}, \forall i$, $\forall j$ and $\forall k$, in terms of $r$ and $S(r)$. Substituting these into (1) and rearranging it as

$$
\begin{aligned}
M= & {\left[(1+\mu)\left(1+\frac{\widehat{m}}{m} p\left(1-\widehat{m}^{w}\right)\right)\right] r+\left[\left[\frac{(1+\gamma) v+\sigma}{\sigma}+\mu\right] \widehat{m}^{x} \phi\right.} \\
& \left.\left.+(1+\mu)\left[\phi+m^{-1}\left(\widehat{m}^{w}+\left(1-\widehat{m}^{x}\right) \widehat{m} \phi-\widehat{m}^{z}\right)\right)\right]\right] S(r)
\end{aligned}
$$

in which $\phi=\widehat{m}^{z} /\left(1-\gamma \widehat{m}^{x+1}\right)$. The expected input to the system, $S(r)$, can be expressed as $S(r)=L_{t} f(r)$, where $f(r)$ indicates the mean number of successfully received PNcodes on a slot-subchannel given $r$ SSs.

$$
f(r)=\sum_{k=0}^{r} \sum_{j=0}^{k} P_{d}(j) \phi_{c}\left(j ; N_{c}\right)\left(\begin{array}{l}
k \\
j
\end{array}\right)\left(1 / L_{t}\right)^{j}\left(1-1 / L_{t}\right)^{k-j}\left(\begin{array}{l}
r \\
k
\end{array}\right) \widetilde{p}^{k}(1-\widetilde{p})^{r-k}
$$

with $\tilde{p}=p(1-m)$. We denote the mean number of the distinct PN codes transmitted on a slot-subchannel, provided that $j$ SSs transmit PN codes randomly chosen among total of $N_{c}$ PN codes by $\phi_{c}\left(j ; N_{c}\right)$, which is given in [3]. $P_{d}(j)$ is the probability that a PN code sent by an SS will be successfully identified among $j$ codes in a slot-subchannel given $N_{b}$ neighboring BSs, which is heuristically expressed as $P_{d}(j)=\left(e^{\theta\left(j-\theta_{t}\right)}+\varepsilon \sqrt{N_{b}}\right)^{-1}$, where the first and second terms in the right-hand side represent intra- and inter-cell multiple access interference(MAI), respectively. The parameter, $\theta$, is the extent of degradation due to MAI and $\theta_{t}$ is threshold at which $P_{d}(j)$ is $1 / 2$, when $\varepsilon=N_{b}=1$. The parameter, $\varepsilon$, is a weight for intercell MAI. The second term must be greater than or equal to $1 . P_{d}(j)$ can be also derived by considering physical parameters [8]. For measuring performance, we define $r_{e}$ as the equilibrium number of $r$ in $R$ state of the system which can be obtained by solving (2). Using Little's result, one can obtain the initial access delay, $\bar{D}$, and access throughput, $\bar{S}$, respectively as follows.

$$
\bar{D}=\left(r_{e}+\sum_{k=0}^{w-1} p_{R k}+\sum_{l=0}^{y-1} \underline{h}_{l}+h\right) / \bar{S}
$$

with $\bar{S}=S\left(r_{e}\right)$. Whenever unsuccessful accesses occur, each SS involved increases its contention window size in a binary exponential manner. That is, the contention window size after the $k$-th collision, $W(k)$, is given by

$$
W(k)=\min \left(W_{0} 2^{(k-1)}, W_{m}\right), \quad 1 \leq k \leq K_{m}
$$

where $W_{0}$ is an initial window size and $W_{m}$ is the maximum of $W(k)$. When $W(k)$ reaches $W_{m}, W_{m}$ is repeatedly used. An SS deferes its retransmission the time randomly selected among $W(k)$. In order to include a binary exponential backoff algorithm, we estimate the retransmission probability, $p$, as follows. With an initial guess for $p$ and $r_{e}$, one can obtain the transmission success probability, $p_{s}$, as follows. 


$$
\bar{W}=\sum_{k=1}^{K_{m}} p_{s}\left(1-p_{s}\right)^{k}\left(\frac{W(k)-1}{2}\right), \text { with } p_{s}=S\left(r_{e}\right) / r_{e}
$$

where $K_{m}$ is the maximum exponent of $W(k)$. Then, the retransmission probability, $p$, can be obtained by $p=1 / \bar{W}$. By updating, and substituting $p_{s}$ and $p$ into (2) at each iteration, we can obtain $\bar{S}$ and $\bar{D}$ when both $p$ and $r_{e}$ converge.

\section{Numerical Examples}

The parameters of $P_{d}(j), \theta=1.5, \theta_{t}=4.5, \varepsilon=0.42$ and $N_{b}=6$ are used. For delay parameters, $x=3, y=8, z=3$ and $w=5$ are also used with $p_{h}=0.8$. Additionally, the parameters of the binary exponential backoff algorithm, $W_{0}=1, N_{m}=6$ and $W_{m}=70$, are used. Finally, the piggyback probability, $\gamma$, number of PN codes, $N_{c}$, number of slot-subchannels, $L_{t}$, and the traffic generation probability, $\sigma$, are respectively set to $0.01,4$ and 6 .

In Figs. 3 and 4, by varying $\mathrm{HO}$ occurrence probability, $m$, HO ranging success probability, $p_{h}$, and the population size, $M, \bar{S}$ and $\bar{D}$ are respectively depicted. When $m=0$,

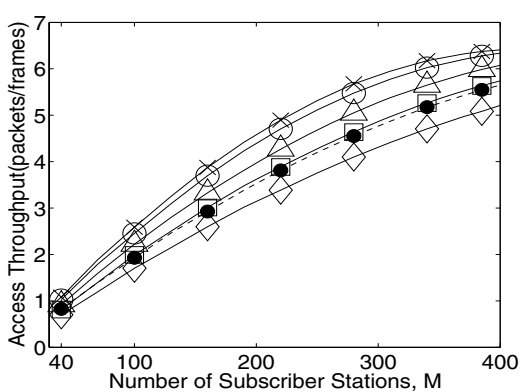

Fig. 3. Access Throughput $(y=8, m=$ $[\times] 0.001,[\circ] 0.01,[\triangle] 0.03,[\square] 0.05,[\diamond]$ $0.08,[\bullet] y=5, m=0.08)$

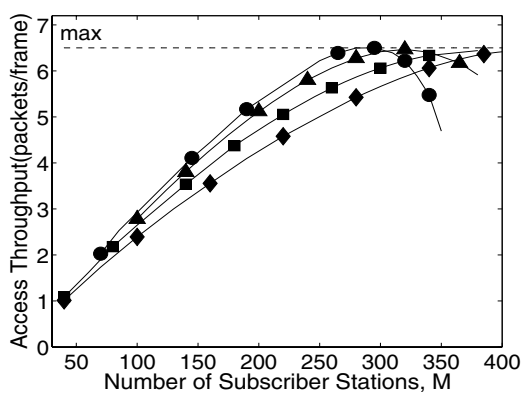

Fig. 5. Access Throughput $(m=0.01, \sigma=0.04$, $\left.W_{0}=5, W_{m}=[\bullet] 8,[\mathbf{\Delta}] 16,[\mathbf{\bullet}] 32,[\bullet] 64\right)$

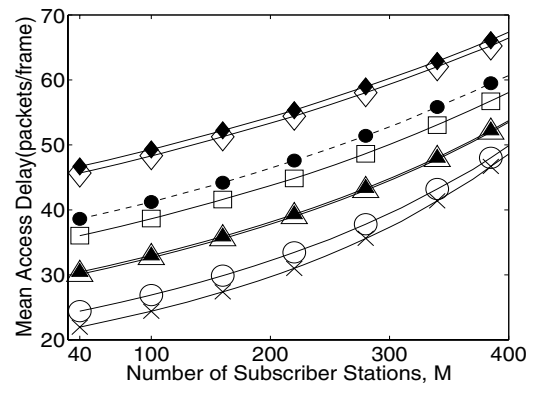

Fig. 4. Mean Access Delay $\left(y=8, p_{h}=0.8\right.$, $m=[\times] 0.001,[0] 0.01,[\triangle] 0.03,[\square] 0.05$, $[\diamond] 0.08,[\bullet] y=5, m=0.08 ; p_{h}=0.6$, $m=[\bullet] 0.03,[\diamond] 0.08)$

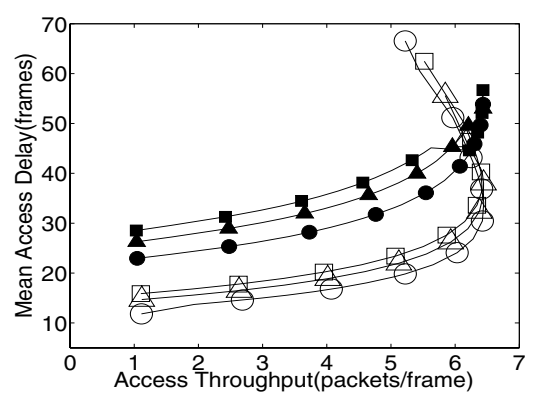

Fig. 6. Mean Access Delay vs. Access Throughput $\left(m=0.01, \quad \sigma=0.04 ; \quad W_{m}=32\right.$, $W_{0}=[\circ] 1,[\triangle] 3,[\square] 5 ; W_{m}=64, W_{0}=[\bullet] 1$, $[\mathbf{\Delta}] 3,[\mathbf{\square}] 5)$ 
the model just considers the performance of IEEE 802.16d random access protocol, which doesn't include handover procedure. When $m$ becomes large, $\bar{S}$ and $\bar{D}$ respectively decreases and increases. This can be expected, because SSs with high mobility will more frequently experience the handover procedure which includes its own delay and the retransmission procedure, compared to SSs with low mobility. In addition, when handover signalling delay, $y$, is reduced, $\bar{S}$ and $\bar{D}$ respectively increases and decreases. In Figs. $5, \bar{S}$ is observed according to $W_{m}$. When $W_{m}$ increases, the maximum of $\bar{S}$ moves toward large $M$. In Fig.6, $\bar{S}$ is depicted versus $\bar{D}$.

\section{Conclusion}

In this paper, we examined the performance of random access protocol of IEEE802. 16e with handover procedure, in terms of access throughput and mean access delay as an extention of [3]. The increase of mobility results in the reduction of access throughput and the increase of mean access delay, which results from the fact that each handover process includes the random access procedure as well as its own signalling delay. Although EPA provides accurate results for a stable system with large number of stationary SSs in a BS [7, as a future work, the analytical results may be validated by simulation according to handover occurrence probability, due to variance of number of SSs handed over among BSs. It would be also interesting to analyze the performance when an SS's mobility shows memory.

\section{Acknowledgement}

This work was supported by grant No.B1220-0501-0232(2005) from the University fundamental Research Program of the Ministry of Information \& Communication in Republic of Korea.

\section{References}

1. Draft IEEE Standard for Local and metropolitan area networks, Part 16: Air Interface for Broadband Wireless Access Systems(IEEE802.16 REVd/D5-2004), May, 2004.

2. Draft IEEE Standard for Local and metropolitan area networks, Part 16: Air Interface for Broadband Wireless Access Systems-Amendment for Physical and Medium Access Control Layers for Combined Fixed and Mobile Operation in Licensed Bands(IEEE P802.16e/D7), April, 2005.

3. Jun-Bae Seo, Nam-Suk Lee, Nam-Hoon Park, Hyong-Woo Lee and Choong-Ho Cho, "Performance Analysis of IEEE802.16d Random Access Protocol," Accepted for publication at IEEE ICC'06, Available on Request.

4. Jeong-Jae Won, Choong-Ho Cho, Hyong-Woo Lee and Victor Leung, "Stabilization of Contention-Based CDMA Ranging channel in Wireless Metropolitan Area Networks," in Proc. of Networking 2005, LNCS vol.3462, pp.1255-1266, Waterloo, Canada, May 2-6, 2005.

5. Hyun-Hwa Seo, Byung-Han Ryu, Hyong-Woo Lee and Choong-Ho Cho, "Design of Performance Analysis Model for Efficient Random Access Protocol in CDMA based OFDMA-PHY System," IEEE ICACT'05, vol.1, pp.347-351, 2005. 
6. Hyun-Hwa Seo, Choong-Ho Cho, Hyong-Woo Lee, "Traffic Characteristics based Performance Analysis Model for Efficient Random Access in OFDMA-PHY System," in Proc. of WWIC 2005, LNCS vol.3510, pp.213-222, Xanthi, Greece, May 11-13, 2005.

7. Shuji Tasaka, Performance Analysis of Multiple Access Protocols, MIT Press, 1986.

8. Jisang You, Kanghee Kim and Kiseon Kim, "Capacity evaluation of the OFDMA-CDMA ranging subsystem in IEEE802.16-2004,” in Proc. of IEEE WiMob’05, vol.1, pp.100-106. 